\title{
Evaluación del nicho ecológico de formas larvales de Aedes aegypti y Culex quinquefasciatus (Diptera: Culicidae) en una comunidad urbana de San José, Costa Rica
}

\author{
OLGER CALDERÓN-ARGUEDAS* y ADRIANA TROYO****
}

\begin{abstract}
EVALUATION OF THE ECOLOGICAL NICHE OF Aedes aegpti AND Culex quinquefasciatus (DIPTERA: CULICIDAE) LARVAE IN AN URBAN COMMUNITY OF SAN JOSÉ, COSTA RICA
\end{abstract}

Observations related to the width and overlap of the ecological niche for larval forms of Aedes aegypti and Culex quinquefasciatus in artificial containers were performed using the data obtained during two laval surveys in the four sectors of the neighborhood "La Carpio", San José, Costa Rica. "La Carpio" was the first community of the Metropolitan Area of San José that suffered a dengue outbreak. The first survey was performed in the dry season of 2003 and the second one in the wet season of the same year. The width of niche was expressed using the Levin Index, and the overlap of niche was determined by the Morisita Index modified by Horn. In both larval surveys Ae. aegypti and $C x$. quinquefasciatus were present. Ae. aegypti showed higher values for width of niche in the four sectors of the neighborhood and during both surveys.During the first larval survey (dry season of 2003), there was an overlap of niche only in sectors 1 and 3. In the wet season, the overlap was observed in the four sectors, but the values were higher in sectors 1 and 2. Results show that Ae. aegypti is the most common species of mosquitoes in artificial containers in the neighborhood, and that the role of $\mathbf{C x}$. quinquefasciatus as modulator of the Ae. aegypti populations could be limited.

Key words: Aedes aegypti, Culex quinquefasciatus, Culicidae-ecological niche, Costa Rica.

\section{INTRODUCCIÓN}

Aedes aegypti y Culex quinquefasciatus constituyen dos de las especies de mosquitos más comunes en los ecosistemas urbanos ${ }^{1}$. Ambas especies han sido vinculadas con la transmisión de organismos patógenos. En el caso de Ae. aegypti, el virus del dengue en sus cuatro serotipos, así como el virus de la fiebre amarilla son los principales agentes etiológicos transmitidos por el mosquito ${ }^{2}$. Para $\mathrm{C} x$. quinquefasciatus, parasitosis como la filariasis de Bancroft y virosis como la encefalitis equina de San Luis y la encefalitis por el Virus del Nilo Occidental (WNV) figuran entre las principales enfermedades infecciosas de las cuales esta especie se desempeña como vector ${ }^{2}$.

Algunos estudios han podido demostrar que las formas larvales de estas dos especies de mosquitos pueden compartir eficientemente el

\footnotetext{
* Centro de Investigación en Enfermedades Tropicales (CIET), Departamento de Parasitología, Facultad de Microbiología, Universidad de Costa Rica. San José, Costa Rica.

** Global Public Health Program, Department of Epidemiology and Public Health, University of Miami, FL, USA.
} 
hábitat ${ }^{3}$. También se ha podido advertir que cuando se da la eliminación de alguna de éstas, la otra realiza una ocupación efectiva del hábitat abandonado ${ }^{1,3}$. En este sentido surge la necesidad de valorar los aspectos relacionados con el nicho ecológico de cara a poder perfilar y optimizar acciones de prevención y control para estas especies.

De acuerdo a Pianka, la amplitud del nicho no es más que la suma total de la variedad de recursos diferentes utilizados por una unidad organismal, lo que puede ser interpretado como una medida de especialización ${ }^{4}$. El solapamiento ocurre cuando dos o más organismos usan una porción de un mismo recurso simultáneamente. Este recurso puede estar constituido por los sustratos tróficos requeridos por las especies que comparten el nicho ${ }^{5}$. Los valores matemáticos que se le asignan al solapamiento de nicho fluctúan entre 0 (cuando no tiene lugar ningún solapamiento) y 1,00 (solapamiento completo) ${ }^{6}$. El solapamiento de nicho estima la competencia por recursos entre dos o más organismos ${ }^{3}$.

Ae. aegypti y $C x$. quinquefasciatus constituyen las especies más frecuentes de mosquitos en contenedores artificiales en el caserío La Carpio, San José, Costa Rica ${ }^{7}$. En el presente estudio se pretendió realizar una evaluación cuantitativa de la amplitud y el solapamiento del nicho ecológico, con el fin de visualizar la dominancia de las especies, así como sus posibles vínculos en lo referente a modulación de los tamaños poblacionales larvales, en la comunidad la Carpio, la cual constituyó la primera comunidad del Área Metropolitana de San José, Costa Rica en sufrir un brote de dengue.

\section{MATERIAL Y MÉTODOS}

"La Carpio" constituyó el escenario de varios estudios en donde se han abordado diversos aspectos relativos a una iniciativa de participación comunitaria $^{7-9}$.

Los datos concernientes a dos de las cuatro encuestas entomológicas que se practicaron en el lugar (datos en publicación) fueron utilizados para realizar la evaluación relativa a la amplitud y el solapamiento de nicho para Ae. aegypti y $C x$. quinquefasciatus. Las encuestas consideradas fueron las que tuvieron lugar en las estaciones seca y lluviosa del año 2003. En estas encuestas se colectaron datos sin que hubiese mediado ninguna acción de intervención por parte de la comunidad por lo cual pueden reflejar las condiciones ecológicas usuales del lugar.

La descripción de la comunidad y los protocolos de ejecución de las encuestas larvales fueron dados a conocer previamente ${ }^{9}$. Los tipos de contenedores analizados en cada encuesta y sobre los cuales se hicieron los cálculos relativos a la amplitud y el solapamiento de nicho fueron baldes, barriles, llantas, floreros, macetas, latas, pilas, orificios fijos y objetos misceláneos.

En el presente estudio se estimó la amplitud del nicho ecológico mediante el índice de Levin ${ }^{3}$, el cual consiste en lo siguiente:

\section{Amplitud de Nicho: $B^{\prime}=1 / \Sigma p_{i}^{2}$}

El solapamiento de nicho fue calculado mediante el Índice de Morisita (1959) ajustado por Horn ${ }^{10}$ : $\Sigma \mathrm{p}_{\mathrm{ik}}{ }^{2}$

Solapamiento de Nicho: $\mathrm{Ci}=2 \Sigma \mathrm{p}_{\mathrm{ij}} \mathrm{p}_{\mathrm{ik}} / \Sigma \mathrm{p}_{\mathrm{ij}}^{2}+$

En ambos casos $\mathrm{P}_{\mathrm{i}}$ representa la proporción de recursos utilizados por la población larval respectiva.

\section{RESULTADOS}

Aedes aegypti y $C x$. quinquefasciatus fueron observados en ambas encuestas entomológicas (Tablas 1-3). Tanto en la estación seca como la lluviosa, Ae. aegypti presentó los valores más elevados en lo que respecta a los índices de amplitud de nicho. En la estación seca, para Ae. aegypti, estos índices tuvieron valores entre 1,86 (Sector 3) y 2,77 (Sector 1); mientras que en la estación lluviosa los mismos mostraron valores entre 2,88 (Sector 3) y 6,66 (Sector 4) (Tabla 1). Para $C x$. quinquefasciatus los índices de amplitud de nicho en la estación seca estuvieron entre 1,00 (Sectores 1,2 y 3 ) y 1,81 (Sector 4), mientras que en la estación lluviosa los mismos fluctuaron entre 1,00 (Sector 3) y 2,00 (Sector 2) (Tabla1).

En relación con el solapamiento de nicho, durante la estación seca, dicho solapamiento se pudo documentar sólo en los sectores 1 y 3 y los valores respectivos fueron 0,58 (Sector 1) y 0,78 (Sector 3) (Figura 1). En la estación lluviosa, el solapamiento fue observado en todos los 
Tabla 1. Amplitud de nicho para Aedes aegypti y Culex quinquefasciatus estimado mediante el Índice de Levin, en las cuatro encuestas entomológicas en "La Carpio"

\begin{tabular}{|c|c|c|c|c|c|c|c|c|}
\hline \multirow[t]{3}{*}{ Encuesta } & \multicolumn{8}{|c|}{ Sector } \\
\hline & \multicolumn{2}{|r|}{1} & \multicolumn{2}{|c|}{2} & \multicolumn{2}{|c|}{3} & \multicolumn{2}{|c|}{4} \\
\hline & $\begin{array}{c}\text { Ae. } \\
\text { Aegypti }\end{array}$ & $\begin{array}{c}C x . \\
\text { quinque- } \\
\text { fasciatus }\end{array}$ & $\begin{array}{c}\text { Ae. } \\
\text { aegypti }\end{array}$ & $\begin{array}{c}C x . \\
\text { quinque- } \\
\text { fasciatus }\end{array}$ & $\begin{array}{c}\text { Ae. } \\
\text { aegypti }\end{array}$ & $\begin{array}{c}\text { Cx. } \\
\text { quinque- } \\
\text { fasicatus }\end{array}$ & $\begin{array}{c}\text { Ae. } \\
\text { aegypti }\end{array}$ & $\begin{array}{r}\text { Cx. } \\
\text { quinque- } \\
\text { fasciatus }\end{array}$ \\
\hline $1(\mathrm{Se}$ & 2,77 & 1,00 & 2,0 & 1,00 & 1,86 & 1,00 & 2,13 & 1,81 \\
\hline 2 (Lluviosa 2003) & 3,03 & 1,82 & 3,07 & 2,00 & 2,88 & 1,00 & 6,66 & 1,00 \\
\hline
\end{tabular}

sectores y los índices respectivos fluctuaron entre 0,09 (Sector 3) y 0,86 (Sector 2) (Figura. $1)$.

Durante la estación seca el mayor solapamiento tuvo lugar en baldes en tanto que en la estación lluviosa dicho solapamiento se dio en baldes y llantas.

En la estación seca los contenedores mayoritariamente positivos por larvas, indistintamente de la especie, fueron los baldes y las macetas (Tabla 2). Mientras que en la estación lluviosa los baldes y barriles figuraron como depósitos donde fue frecuente encontrar larvas de Ae. aegypti y $C x$. quinquefasciatus. Sin embargo, para Ae. aegypti los objetos misceláneos, agrupados en la categoría de "otros", constituyeron los principales sitios de oviposición y desarrollo larval en esta estación (Tabla 3).

\section{DISCUSIÓN}

Ae. aegypti es un mosquito adaptado a vivir en el ambiente intra y peridomiciliar. Su limitada capacidad de dispersión así como su dependencia trófica del ser humano en su fase adulta condicionan su sinantropismo ${ }^{11}$.

En Costa Rica, la reintroducción de Ae. aegypti tuvo lugar inicialmente durante los años setenta en algunas áreas costeras del país. Para los primeros años de la década de los noventa esta especie se había convertido en el principal mosquito en ambientes urbanos y peri urbanos a lo largo de toda la geografía nacional ${ }^{12}$.

En el presente estudio se pudo constatar que tanto en la estación seca como en la lluviosa Ae. aegypti presentó valores de amplitud de nicho superiores a los de $C x$. quinquefasciatus, su más inmediato competidor en lo que respecta a la utilización de depósitos de agua artificiales para realizar su oviposición (Tablas 1-3). Esto refleja el amplio espectro de recursos que esta especie puede utilizar como sitios de oviposición y desarrollo larval.

No obstante, el efecto de las lluvias fue evidente, razón por la cual los valores de amplitud de nicho para ambas especies fueron superiores en la segunda encuesta larval con respecto a la primera. En la época lluviosa y para este tipo de comunidades, la gran cantidad de potenciales hábitats larvales de ubicación peridomiciliar, que usualmente se acumulan como resultado de un mal manejo de desechos sólidos, se llenan con agua, por lo que se convierten en criaderos efectivos para estas y otras especies de mosquitos. Este efecto fue patente en Ae. aegypti (Tabla 3). La abundancia de recursos apropiados para la oviposición promueve un incremento en la magnitud de las poblaciones de estos dípteros.

Durante la estación seca los contenedores usados para el almacenamiento de agua como por ejemplo los baldes y barriles constituyen los depósitos preferenciales donde los mosquitos realizan su oviposición. A estos hay que agregar los floreros que usualmente mantienen poblaciones larvales que pasan desapercibidas por los habitantes de las viviendas. Aunque usualmente en esta época las densidades poblacionales de los mosquitos suelen ser muy bajas, este tipo de depósitos son capaces de mantener poblaciones basales que serán las que sirvan para el disparo poblacional que ocurren en las estaciones lluviosas.

Las diferencias observadas en los índices de amplitud en cada una de las especies pueden obedecer a condiciones particulares en cada uno de los sectores que condicionarían la utilización de diferentes tipos de recursos disponibles para la oviposición (Tablas 2-3).

En relación con el solapamiento de nicho se pudo ver que los contenedores de mediano 


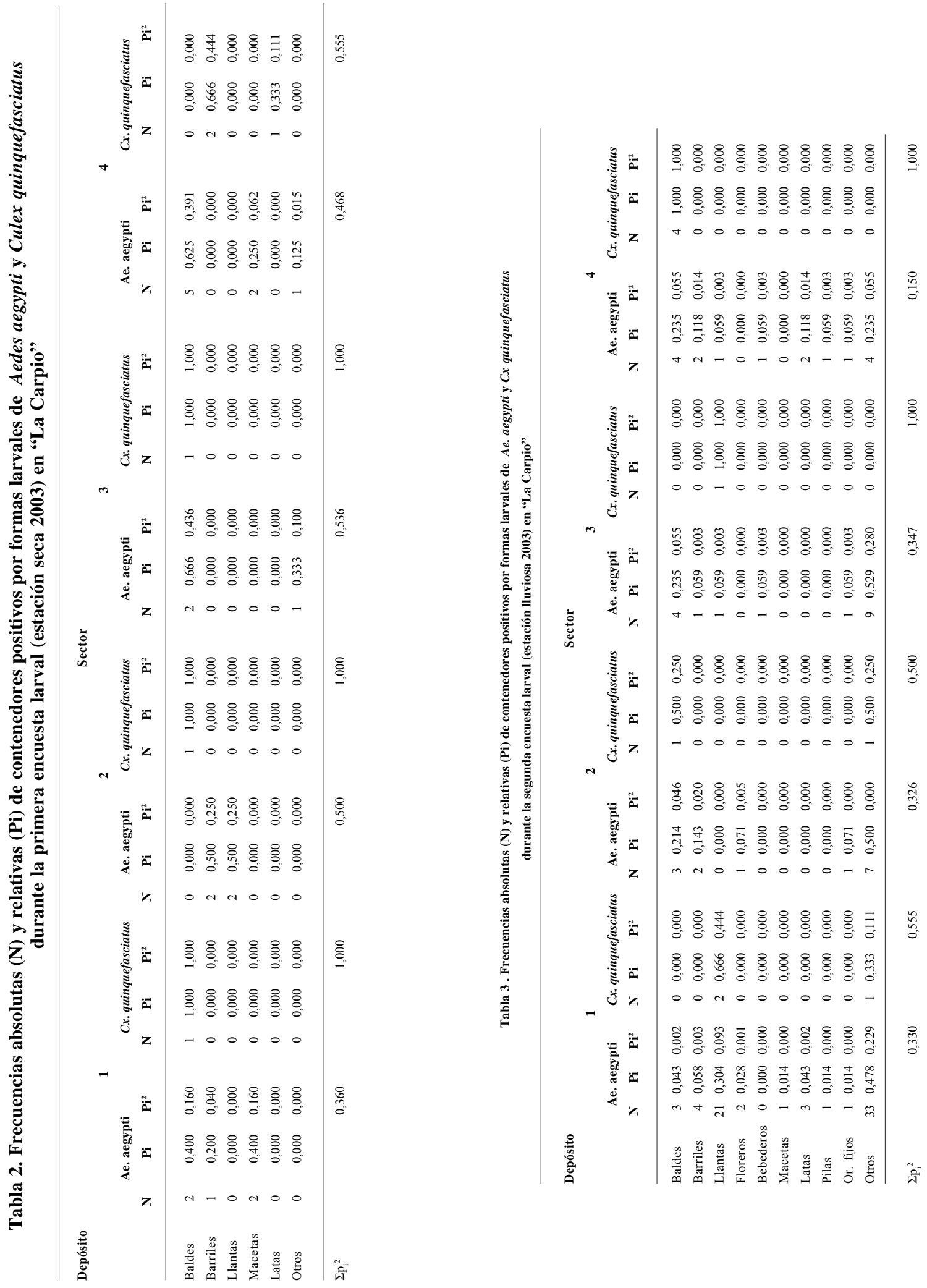


tamaño como baldes y llantas constituyeron el principal escenario para que éste se diera (Tablas 2 y 3). Esta situación es similar a la descrita en Italia $^{13}$, en la cual Aedes albopictus y Culex pipiens mostraron su mayor solapamiento en contenedores representados por baldes y orificios. Al igual que lo expuesto anteriormente en lo referente a amplitud de nicho, las lluvias produjeron un efecto positivo en relación con el solapamiento de nicho. En la estación seca, solamente en dos sectores pudo evidenciarse el solapamiento, lo que podría obedecer a la limitada cantidad de depósitos adecuados disponibles para la oviposición y las bajas poblaciones vectoriales. Cabe destacar que los sectores 1 y 3 , donde el solapamiento tuvo lugar, son sectores colindantes en términos geográficos, lo que supone la ocurrencia de características urbano ambientales semejantes. En algunos estudios y en otras geografías se ha demostrado que durante la estación seca es más bien cuando tiene lugar el mayor solapamiento de nicho entre formas larvales de los mosquitos urbanos ${ }^{13}$. Esta situación se relaciona con una clara reducción en el número de depósitos disponibles para la oviposición lo que supone una marcada competencia por estos recursos. Este fue el caso descrito en el estudio referido anteriormente en el cual tuvo lugar un alto solapamiento entre Ae. albopictus y $C x$. pipiens durante los meses de verano en la localidad Desenzano del Garda, Italia ${ }^{13}$.

En la estación lluviosa, los cuatro sectores mostraron solapamiento de nicho entre $A e$. aegypti y $C x$. quinquefasciatus con sus diferencias particulares de acuerdo al sector. En dos de los sectores, los índices de solapamiento fueron superiores a 0,80 (Figura. 1) lo que demuestra que pueden darse infestaciones mixtas estables, como ha sido previamente demostrado ${ }^{7}$ y que el eventual papel regulador de $C x$. quinquefasciatus respecto a las poblaciones de Ae. aegypti prácticamente no se da, por lo que Ae. aegypti se mantiene dominante en el poblado. En estudios realizados en Güines, provincia de la Habana, Cuba, donde las campañas de control del Ae. aegypti han sido exitosas, se ha podido constatar que aunque la frecuencia de infestación por Ae. aegypti es muy baja en comparación con $C x$. qunquefasciatus, su inmediato competidor, el solapamiento de nicho entre ambas especies suele ser alto, lo que indica que en este ecosistema urbano en particular ambas especies comparten

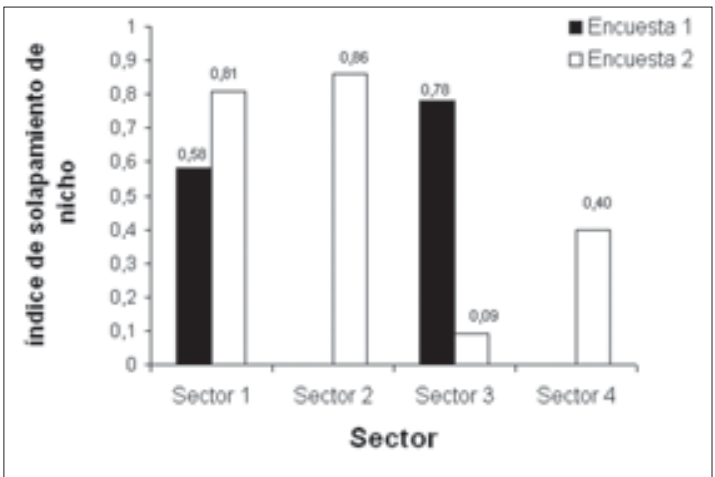

Figura 1. Índice de Morisita ajustado por Horn, para la estimación del solapamiento de nicho entre Aedes aegypti y Culex quinquefasciatus durante los cuatro ciclos de muestreo.

un gran número de recursos ${ }^{3}$.

Dado que el efecto regulador del solapamiento de nicho no parece darse entre las especies estudiadas, las acciones preventivas que afectarían ambas especies deben complementarse con acciones de efecto individual. En el caso de Ae. aegypti una adecuada remoción o tratamiento de los depósitos de agua intra y peridomiciliares constituyen el más efectivo mecanismo para controlar las poblaciones y disminuir el riesgo de transmisión del dengue. En el caso de $C x$. quinquefasciatus, las anteriores medidas podrían disminuir la infestación domiciliar por este díptero, pero considerando que $C x$. quinquefasciatus puede utilizar como sitios de desarrollo que no necesariamente son resultado del sinantropismo, el manejo integrado de los ambientes peri urbanos como complemento a las acciones contra el Ae. aegypti, resultarían el método efectivo para establecer su control.

El conocimiento de las relaciones ecológicas entre las especies de mosquitos vectores que se establecen en ecosistemas particulares, es un insumo de gran importancia para el desarrollo, planificación y optimización de acciones de prevención y control para estas especies y para los patógenos que transmiten.

\section{RESUMEN}

Se realizaron observaciones relativas a la amplitud y solapamiento del nicho ecológico para las formas larvales de Aedes aegypti y Culex quinquefasciatus en contenedores artificiales, 
utilizando datos obtenidos in dos encuestas larvales que se ejecutaron en los cuatro sectores que conforman el poblado "La Carpio", San José, Costa Rica. "La Carpio" fue la primer comunidad del Área Metropolitana de San José en sufrir un brote de dengue. La primera encuesta tuvo lugar en la estación seca del 2003 y la segunda se realizó en la estación lluviosa del mismo año. La amplitud de nicho fue expresada por medio del Índice de Levin y el solapamiento de nicho fue calculado mediante el Indice de Morisita modificado por Horn. En ambas encuestas Ae. aegypti y $C x$. quinquefasciatus estuvieron presentes. Ae. aegypti mostró los valores más elevados en lo referente a amplitud de nicho en los cuatro sectores de la comunidad durante ambas estaciones. En la primer encuesta larval (estación seca del 2003), hubo solapamiento de nicho sólo en los sectores 1 y 3 . En la estacón lluviosa el solapamiento fue observado en los cuatro sectores, pero los valores más elevados se dieron en los sectores 1 y 2 . Los resultados muestran que Ae. aegypti es el mosquito más abundante en contenedores artificiales en la comunidad y el papel de $C x$. quinquefasciatus como modulador de las poblaciones parece ser limitado.

\section{REFERENCIAS}

1.- BISET J A, MARUETTI M C. Comportamiento de las densidades larvales de Aedes (S.) aegypti y Culex (C.) quinquefasciatus durante la etapa intensiva de la campaña anti-aegypti. Rev Cub Med Trop 1983; 35: 176-80.

2.- HARDWOOD R, JAMES M. Mosquitoes. En: Entomology in human and animal health. $7^{\text {th }}$ edition. The Macmillan Publishing Co. Inc. New Yor, 1979. $548 \mathrm{pp}$.

3.- BISSET-LAZCANO J A, MARQUETTI M C, GONZÁlEZ B, et al. Algunos aspectos del nicho ecológico de Aedes (S.) aegypti (Linnaeus, 1762)
(Diptera: Culicidae) y Culex (C.) quinquefasciatus Say, 1823 (Diptera: Culicidae) en el ambiente urbano. Rev Cub Med Trop 1987; 39: 113-8.

4.- PIANKA E. Competition and niche overlap. A method of measuring them. Ecology 1976; 53: 687-92.

5.- SMITH R L, SMITH T M. Interespecific competition. En: Elements of ecology. The Benjamin/Cummings Publishing Co. CA. 1998. 555 pp.

6.- ABRAMS P. Some comments on measuring niche overlap. Ecology 1980; 61: 44-9.

7.- CALDERÓN-ARGUEDAS O, TROYO A, SOLANO M E. Diversidad larval de mosquitos (Diptera: Culicidae) en contenedores artificiales procedentes de una comunidad urbana de San José, Costa Rica. Parasitol. Latinoam 2004; 59: 132-6.

8.- CALDERÓN-ARGUEDAS O, TROYO A, SOLANO M E. La participación comunitaria como recurso para la prevención del dengue en una comunidad urbana de San José, Costa Rica. Rev Costar Sal Pub 2005; 14: 51-7.

9.- CALDERÓN-ARGUEDAS O, TROYO A, SOLANO M E. Caracterización de los sitios de multiplicación de Ae. aegypti (Diptera: Culicidae) en el caserío "La Carpio", San José, Costa Rica durante la estación seca del año 2003. Rev Biomed 2004; 15: 73-9.

10.- SMITH E P, ZARET T M. Bias in estimating niche overlap. Ecology 1982; 63: 1248-53.

11.- KUNO G. Review of the factors modulating dengue transmission. Epidemiol Rev 1995; 17: 321-35.

12.- TROYO A, PORCELAIN S L, CALDERÓNARGUEDAS O, et al. Dengue in Costa Rica: The gap in local scientific research. Pan Am J Public Health 2006; 20: 350-60.

13.- CARRIERI M, BACCHI M, BELLINI R, MAINI S. On the competition occurring between Ae. albopictus and Culex pipiens (Diptera: Culicidae) in Italy. Env Entomol 2003; 32: 1313-21.

Agradecimientos: Los autores desean externar su agradecimiento al Dr. Oscar Montero, las licenciadas Fredda Whilhem, Carmen Zúñiga y al personal asistencial del EBAIS de la comunidad "La Carpio" por la colaboración prestada. A los doctores Mayra E. Solano, Adrián Avendaño y a los estudiantes que participaron en el Trabajo Comunal TC-492 por su labor operativa. También se agradece a la Sección de Trabajo Comunal Universitario, Vicerrectoría de Acción Social, Universidad de Costa Rica por su apoyo económico y logístico.

Correspondencia a:

E-mail: olgerc@cariari.ucr.ac.cr. 